\title{
Potenciais evocados auditivos de longa latência em adultos com HIV/Aids*****
}

\author{
Long latency auditory evoked potentials in adults with HIV/Aids
}

\author{
Aline Covo da Silva* \\ Fernanda Rodrigues Pinto** \\ Carla Gentile Matas***
}

*Fonoaudióloga. Graduada pela Faculdade de Medicina da Universidade de São Paulo. Endereço para correspondência: Rua Cipotânea, 51 - Campus Cidade Universitária - São Paulo - SP - CEP 05360-160 (aline.covo@uol.com.br)

**Fonoaudióloga.

***Fonoaudióloga. Doutora em Distúrbios da Comunicação Humana pela Universidade Federal de São Paulo - Escola Paulista de Medicina Professora Associada do Curso de Fonoaudiologia da Faculdade de Medicina da Universidade de São Paulo.

****Pesquisa Realizada no Laboratório de Investigação Fonoaudiológica em Potenciais Evocados Auditivos do Curso de Fonoaudiologia da Faculdade de Medicina da Universidade de São Paulo.

Artigo Original de Pesquisa

Artigo Submetido a Avaliação por Pares

Conflito de Interesse: não

Recebido em 11.06.2007.

Revisado em 13.09.2007.

Aceito para Publicação em 29.10.2007.

\begin{abstract}
Background: Long Latency Auditory Evoked Potentials. Aim: to characterize the Long Latency Auditory Evoked Potentials (LLAEP) in individuals with HIV/AIDS in comparison to a control group. Method: the research sample was composed by 21 individuals with HIV/AIDS - research group (14 male and 7 female), with ages ranging from 31 to 48 years, and 21 healthy individuals - control group (5 male and 16 female), with ages ranging from 19 to 36 years. The latency and amplitude values of the P300 wave were analyzed; latency of N1 and P2 waves, and amplitude N1-P2. The electrodes were placed on the following positions: A1, A2, Cz and Fpz. Results: the T-student test was used to analyze the results and the adopted significance level was of 5\%. In the analyzes of P300 it was observed that the group with HIV/AIDS presented greater latency values (p-value $=0,010$ ) and lower amplitude values ( $\mathrm{p}$-value $=0,021$ ) when compared to the control group. The analysis of the N1-P2 complex revealed that the research group presented higher latency values for both, N1 wave (p-value $=0,035)$ and P2 wave, however for this last one, there was no significant statistical difference when compared to the control group. Concerning the amplitude analysis of the N1-P2 complex, it was verified that the control group presented significantly higher values when compared to the research group. Conclusion: the findings of this study indicates that individuals with HIV/AIDS present alterations in the Long Latency Auditory Evoked Potentials (higher latencies and lower amplitudes of N1, P2 and P300 waves), suggesting a disorder in the cortical regions of the auditory pathway, and therefore stressing the importance of such tests in the evaluation of these individuals.
\end{abstract}

Key Words: HIV; Event-Related Potentials P300; Evoked Potentials; Auditory.

\section{Resumo}

Tema: potenciais evocados auditivos de longa latência. Objetivo: caracterizar os potenciais evocados auditivos de longa latência (PEALL) de indivíduos com HIV/Aids comparando com os obtidos no grupo controle. Método: a casuística foi composta por 21 indivíduos com HIV/Aids pertencentes ao grupo pesquisa (14 do gênero masculino e sete do gênero feminino) com idade entre 31 e 48 anos e 21 indivíduos saudáveis pertencentes ao grupo controle (cinco do gênero masculino e 16 do gênero feminino) com idade entre 19 e 36 anos. Foram analisados os valores de latência e amplitude da onda P300, latência das ondas N1 e P2 e amplitude N1-P2. Os eletrodos foram colocados nas posições A1, A2, Cz e Fpz. Resultados: no P300 observou-se que o grupo com HIV/Aids apresentou maiores valores de latência ( $\mathrm{p}$-valor $=0,010)$ e menores de amplitude ( $\mathrm{p}$-valor $=0,021)$ quando comparados com o grupo controle. Na análise do complexo N1-P2, ao comparar os grupos, verificou-se que o grupo pesquisa apresentou maiores valores de latência tanto para a onda N1 (p-valor $=0,035$ ) como para a onda $\mathrm{P} 2$, porém esta última sem diferença estatisticamente significante. Com relação à análise da amplitude N1-P2, verificou-se que o grupo controle apresentou maiores valores, sendo esta diferença estatisticamente significante quando comparada ao grupo pesquisa. Conclusão: os achados do presente estudo mostraram que indivíduos com HIV/Aids apresentam alterações nos PEALL, sugerindo comprometimento nas áreas corticais do sistema auditivo e mostrando a importância destes testes na avaliação audiológica de indivíduos com HIV/Aids.

Palavras-Chave: HIV; Potencial Evocado P300; Potenciais Evocados Auditivos.

Referenciar este material como: 


\section{Introdução}

O vírus da imunodeficiência humana (Human immunodeficiency virus - HIV) é um retrovírus específico responsável pela síndrome da imunodeficiência adquirida (Acquired immunodeficiency syndrome - Aids), que compromete progressivamente o sistema imunológico, propiciando a ocorrência de diversas infecções oportunistas.

De acordo com a literatura, a incidência de alteração auditiva em pacientes com HIV/Aids pode variar aproximadamente entre 20 e $40 \%$, sendo a perda auditiva decorrente de alterações de orelha externa, orelha média e/ou orelha interna ${ }^{(1-4)}$.

Estudos também demonstram que com o avanço da doença, ocorre um comprometimento progressivo do Sistema Nervoso Central (SNC), incluindo o sistema nervoso auditivo central (SNAC), pela ação direta do vírus sobre as estruturas do SNC ou decorrente de infecções oportunistas ${ }^{(1,5-6)}$.

O sistema nervoso auditivo pode ser avaliado por técnicas comportamentais e eletrofisiológicas, sendo que nas técnicas comportamentais são avaliados aspectos como tipo e grau de alteração das habilidades auditivas, enquanto que nas eletrofisiológicas (potenciais evocados auditivos) avaliam-se a fisiologia e o local da lesão ou disfunção (7). Tanto os estudos que avaliam o processamento auditivo quanto os que avaliam a atividade eletrofisiológica demonstram anormalidades em indivíduos com HIV/Aids ${ }^{(3,5,8-11)}$.

As anormalidades eletrofisiológicas nos potenciais evocados auditivos de tronco encefálico (PEATE) e potencial cognitivo (P300) podem ser encontradas precocemente ${ }^{(3,5,8-11)}$, antes do aparecimento clínico de sintomas como comprometimento neurológico e déficits cognitivos, que fazem parte do complexo demencial da Aids ${ }^{(12-13)}$.

A pesquisa dos potenciais evocados auditivos de longa latência (PEALL) tem se mostrado um método efetivo na investigação do SNAC, em especial do processamento auditivo da informação, isso porque a captação desses potenciais reflete a atividade cortical envolvida em habilidades de discriminação, integração e atenção ${ }^{(14)}$.

Os PEALL têm como componentes: N1 (N100), P2 (P200), N2 (N200), P3 (P300). São observados entre 80 e 700 milissegundos (ms) após a apresentação de um estímulo acústico ${ }^{(15)}$. Esses subdividem-se em potenciais exógenos (N1, P2, $\mathrm{N} 2$ ), fortemente influenciados pelas características físicas do estímulo (intensidade e freqüência, entre outras), e potenciais endógenos (P300), influenciados predominantemente por eventos internos relacionados às habilidades cognitivas.

Alguns autores ${ }^{(16)}$ relatam que as ondas N1 e P2 podem ser utilizadas para examinar o processamento cortical auditivo, como a habilidade para discriminar sons com base em suas propriedades acústicas ou fonéticas, podendo ser úteis na avaliação da integridade auditiva central.

O objetivo do presente estudo foi caracterizar os potenciais evocados auditivos de longa latência em indivíduos com HIV/Aids, comparando com os achados obtidos no grupo controle.

\section{Método}

O presente estudo foi desenvolvido no Laboratório de Investigação Fonoaudiológica em Potenciais Evocados Auditivos do Curso de Fonoaudiologia, do Departamento de Fisioterapia, Fonoaudiologia e Terapia Ocupacional da Faculdade de Medicina da Universidade de São Paulo, cujo projeto recebeu parecer favorável da Comissão de Ética para Análise de Projetos de Pesquisa - CAPPesq da Diretoria Clínica do Hospital das Clínicas e da Faculdade de Medicina da Universidade de São Paulo, sob protocolo número 1026/04.

A casuística foi composta por 21 indivíduos com HIV/Aids pertencentes ao grupo pesquisa (14 do gênero masculino e sete do gênero feminino) com idades entre 31 e 48 anos e 21 indivíduos saudáveis pertencentes ao grupo controle (cinco do gênero masculino e 16 do gênero feminino) com idade entre 19 e 36 anos, sendo que os indivíduos com HIV/ Aids foram encaminhados pela Casa da Aids Fundação Zerbini e pelos Serviços de Saúde da Rede Municipal Especializada em DST/Aids da Secretaria Municipal de Saúde de São Paulo.

Os participantes assinaram um termo de consentimento, no qual estavam descritos todos os procedimentos a serem realizados. Após a realização da anamnese foi dado início à avaliação audiológica, composta pelos seguintes procedimentos: inspeção visual do meato acústico externo com o otoscópio da marca Heine, audiometria tonal e vocal, com os audiômetros modelos GSI 61 e GSI 68 da marca Grason-Stadler, e medidas de imitância acústica com o imitanciômetro modelo GSI 33 da marca Grason-Stadler.

Posteriormente foi realizada a avaliação eletrofisiológica da audição para obtenção do PEALL. No P300, o estímulo utilizado foi o tone 
burst apresentado monoauralmente a $75 \mathrm{~dB}$ NA, numa velocidade de apresentação de 1,1 estímulos por segundo, sendo empregado um total de 300 estímulos. Os eletrodos foram posicionados no vértex $(\mathrm{Cz})$, na fronte $(\mathrm{Fpz})$, nas orelhas direita e esquerda (A2 e A1).

Para a obtenção do P300, o indivíduo foi orientado a prestar a atenção aos estímulos raros $(1500 \mathrm{~Hz})$ que apareciam aleatoriamente, dentro de uma série de estímulos freqüentes $(1000 \mathrm{~Hz})$, sendo solicitado que o indivíduo contasse o número de vezes que o evento raro ocorreu ${ }^{(16,17)}$.

Foram analisados os valores de latência e amplitude da onda P300 no traçado correspondente ao estímulo raro, e as latências das ondas N1 e P2 e a amplitude N1-P2 no traçado correspondente ao estímulo freqüente ${ }^{(16)}$.

Para obtenção do PEALL foi feita a limpeza da pele com pasta abrasiva, sendo os eletrodos fixados à pele do indivíduo por meio de pasta eletrolítica $\mathrm{e}$ fita adesiva (micropore), em posições prédeterminadas.

Os valores de impedância dos eletrodos foram verificados, devendo situar-se abaixo de $5 \mathrm{KOhms.}$

O estímulo acústico foi apresentado por um par de fones TDH 39, eliciando as respostas.

$\mathrm{O}$ teste foi realizado em ambiente eletricamente protegido e acusticamente isolado.

\section{Resultados}

Para análise dos resultados foi utilizado o Teste T de Student, com nível de significância de 5\%.

A análise dos dados foi feita por orelha, totalizando 42 orelhas no grupo pesquisa e 42 orelhas no grupo controle.

Analisando a Tabela 1, verifica-se que o grupo com HIV/Aids apresentou maiores valores de latência da onda P300 (p-valor =0,010) e menores valores de amplitude ( $\mathrm{p}$-valor $=0,021$ ) quando comparados ao grupo controle.

$\mathrm{Na}$ análise do complexo N1-P2, ao comparar os grupos, verificou-se que o grupo pesquisa apresentou maiores valores de latência tanto para a onda N1 (p-valor $=0,035)$ como para a onda P2, porém esta última não apresentou diferença estatisticamente significante (Tabela 2).

Na Tabela 2 foi feita à análise da amplitude do complexo N1-P2, e verificou-se que o grupo controle apresentou maiores valores, sendo esta diferença estatisticamente significante ( $\mathrm{p}$-valor $=$ 0,003 ) quando comparada ao grupo pesquisa.

\section{Discussão}

Devido ao comprometimento do SNC, incluindo o SNAC nos pacientes com HIV/Aids, os potenciais evocados auditivos tem sido um importante instrumento nas pesquisas para obtenção de informações sobre o SNC nessa população. De acordo com Picton ${ }^{(18)}$, o aumento da latência ou a diminuição da amplitude das ondas dos potenciais evocados são evidências de problemas clínicos e/ou sub-clínicos.

$\mathrm{Na}$ Tabela 1 foi feita a comparação das latências da onda P300 entre os grupos controle e pesquisa. Pode-se verificar que houve diferença estatisticamente significante entre os grupos, sendo que o grupo controle apresentou menores latências, o que está de acordo com vários estudos (11, 19-23) que também encontraram alterações nas latências do P300 em indivíduos com HIV/Aids.

Para Tartar et al. ${ }^{(22)}$, o P300 é um exame complementar e útil na verificação de alterações cognitivas presentes nos pacientes com HIV/Aids.

TABELA 1. Comparação da amplitude (em microvolts - $\mu \mathrm{v}$ ) e latência (em milissegundos - ms) da onda P300 entre os grupos controle e pesquisa.

\begin{tabular}{c|c|c|c|c|c}
\hline Variável & Grupo & $\mathrm{N}$ & Média & Desvio-Padrão & Significância (P) \\
\hline latência & pesquisa & 42 & 342 & 46,7 & $0,010^{*}$ \\
P300 & controle & 42 & 320,6 & 25,1 & \\
amplitude & pesquisa & 42 & 8,9 & 3,5 & $0,021^{*}$ \\
P300 & controle & 42 & 10,9 & 4,6 & \\
\hline
\end{tabular}

* p-valor estatisticamente significante.

TABELA 2. Comparação das latências das ondas N1 e P2 (em milissegundos - ms) e da amplitude do complexo N1P2 (em microvolts - $\mu$ v) entre os grupos controle e pesquisa.

\begin{tabular}{cccccccc}
\hline Variável & Grupo & $\mathrm{N}$ & Média & Desvio-Padrão & Significância (P) \\
\hline \multirow{2}{*}{ latência N1 } & pesquisa & 42 & 98,8 & 17,1 & $0,035^{*}$ \\
& controle & 42 & 91,9 & 11,8 & \\
\multirow{2}{*}{ latência P2 } & pesquisa & 42 & 167,1 & 20,5 & 0,081 \\
& controle & 42 & 158,3 & 25,3 & \\
amplitude & pesquisa & 42 & 8,2 & 3,5 & $0,003^{*}$ \\
N1P2 & controle & 42 & 10,6 & 3,7 & \\
\hline
\end{tabular}

* p-valor estatisticamente significante. 
Segundo Fein et al. (20) e Polich et al. ${ }^{(21)}$, o atraso da latência da onda $\mathrm{P} 300$ pode estar relacionado à progressão da doença. Birdsall et al. (24) observaram aumento da latência do P300 e até mesmo ausência desta onda em pacientes com HIV positivo sem evidências clínicas de comprometimento neurológico.

Na Tabela 1 pode-se observar que o grupo controle apresentou maiores amplitudes da onda P300 quando comparado com o grupo pesquisa, sendo esta diferença estatisticamente significante. Estes dados estão de acordo com a pesquisa de Chao et al. ${ }^{(25)}$, que também encontraram alterações neste potencial em indivíduos com HIV/Aids. De acordo com Picton ${ }^{(18)}$, a amplitude do P300 não é um parâmetro confiável na comparação deste potencial entre dois grupos, já que a mesma pode sofrer interferência da atenção.

Na Tabela 2 foi feita a comparação das latências das ondas N1 e P2 entre os dois grupos. Pode-se verificar que ocorreu diferença estatisticamente significante apenas para a latência da onda N1, tendo o grupo controle apresentado menores valores. Com relação à latência da onda $\mathrm{P} 2$, esta apresentou também menores valores para o grupo controle, porém não houve diferença estatisticamente significante quando comparada ao grupo pesquisa.

$\mathrm{Na}$ análise da amplitude do complexo N1-P2 na Tabela 2 verificou-se que o grupo controle apresentou maiores valores, sendo esta diferença estatisticamente significante quando comparada ao grupo pesquisa.

Os achados acima descritos concordam com os obtidos por Chao et al. ${ }^{(25)}$, que analisando as ondas $\mathrm{N} 1$ e P2, também verificaram maiores valores de latências e menores valores de amplitude em indivíduos com HIV/Aids.

Os resultados do presente estudo também corroboram os obtidos por Goodin et al. (26) que em sua pesquisa, verificaram atraso na latência das ondas N1, P1, N2 e P300 em indivíduos com HIV positivo. Segundo os autores, essas alterações foram mais evidentes em indivíduos que se apresentavam sintomáticos, ou seja, com Aids.

No estudo de Galicia et al. (27) com ratos infectados pelo HIV, os autores verificaram redução da amplitude do P300 nas primeiras 24 horas após a contaminação, e do sétimo ao vigésimo primeiro dia; os componentes P2 e N2 também se ausentaram e apenas o N1 pode ser observado. Para os autores, estes achados sugerem alterações no processamento cognitivo. Apesar da etiologia da associação entre HIV e demência ainda não ser conhecida, o P300 parece ser um indicador confiável de alterações cognitivas nos pacientes com HIV e alterações neste potencial sugerem danos neuronais.

Os resultados do presente estudo estão de acordo com os achados de Matas et al. ${ }^{(23)}$, que avaliaram oito indivíduos com HIV/Aids por meio do potencial evocado auditivo de média latência e P300 e compararam os resultados com os obtidos no grupo controle. Os autores verificaram um aumento da latência da onda P300 no grupo estudo, dado sugestivo de comprometimento da via auditiva em regiões corticais e déficit no processamento cognitivo da informação auditiva, reforçando a importância de uma investigação minuciosa da função auditiva em indivíduos com Aids.

Chow et al. ${ }^{(28)}$ encontraram, nos pacientes soropositivos para o HIV, alterações progressivas do SNC, inclusive do SNAC. Para os autores, estas alterações podem ser decorrentes tanto da ação direta do vírus como infecções oportunistas.

\section{Conclusão}

Foi encontrada diferença significante do P300 nos indivíduos com HIV/Aids, tanto nos parâmetros de amplitude quanto de latência. No complexo N1-P2, verificou-se amplitude significantemente reduzida e latência aumentada da onda N1 nos indivíduos com HIV/Aids.

A detecção de alterações neurofisiológicas em indivíduos com HIV/Aids é importante visto que, quanto mais sensível o procedimento para detecção precoce dessas mudanças, maior a possibilidade de um tratamento visando reduzir, minimizar ou reverter os déficits cognitivos associados a infecção (22). Nesse sentido, os achados desse estudo enfatizam que os potenciais evocados auditivos são uma ferramenta fundamental para a avaliação do sistema nervoso auditivo central e de grandeimportância na avaliação audiológica de indivíduos com HIV/Aids. 


\section{Referências Bibliográficas}

1. Madriz JJ, Herrera G. Human immunodeficiency virus and acquired immune deficiency syndrome Aids - related hearing disorders. J. Am. Acad. Audiol. 1995;6(5):35864.

2. Chandrasekhar SS, Connelly PE, Brahmbhatt SS, Shah CS, Kloser PC, Baredes S. Otologic and audiologic evaluation of human immunodeficiency virus-infected patients. Am. J. Otolaryngol. 2000;21(1):1-9.

3. Mata Castro N, Yebra BM, Tutor P, Villarreal GLM, Garcia LF. Hearing loss and human immunodeficiency virus infection. Study of 30 patients. Rev. Clin. Esp. 2000;200(5):271-4.

4. Khoza K, Ross E. Auditory function in a group of adults infected with HIV/Aids in Gauteng, South Africa. S. Afr. J. Commun. Disord. 2002;49(4):17-27.

5. Bankaitis AE, Christinensen LA, Murphy G, Morehouse CR. HIV/Aids and auditory evoked potentials. Semin. Hear. 1998;19(2):177-93.

6. Matkin ND, Diefendorf AO, Erenberg A. Children: HIV/ Aids and hearing loss. Semin. Hear. 1998;19(2):143-53.

7. Schochat E. Avaliação eletrofisiológica da audição. In: Ferreira, L. P.; Befi-Lopes, D. M.; Limongi, S. C. O. Tratado de Fonoaudiologia. São Paulo: Roca; 2004. p. 656-68.

8. Birchall MA, Wight RG, French PD, Cockbain Z, Smith SJ. Auditory function in patients infected with the human immunodeficiency virus. Clin. Otolaryngol. $1992 ; 17(2): 117-21$

9. Somma-Mauvais H, Farnarier G. Evoked potentials in HIV infection. Neurophysiol. Clin. 1992;22(5):369-84.

10. Bankaitis AE, Keith RW. Audiological changes in associated with HIV infection. Ear Nose Throat J. 1995;74(5):353-9.

11. Reyes-Contreras L, Silva-Rojas A, Ysunza-Rivera A, Jimenez-Ruiz G, Berruecos-Villalobos P, Romo-Gutierrez G. Brainstem auditory evoked response in HIV - infected patients with and without Aids. Arch. Med. Res. 2002;33(1):25-8

12. Smith T, Jakobsen J, Gaub J, Helweg-Larsen S, Trojaborg W. Clinical and electrophysiological studies of human immunodeficiency virus - soropositive men without Aids. Ann Neurol. 1988;23:295-7.

13. Ollo C, Johnson R, Grafman J. Signs of cognitive change in HIV disease: an event-related brain potential study. Neurology. 1991;41:209-15.

14. Kraus N, Mcgee T. Potenciais evocados auditivos de longa latência. In: Katz, J. Tratado de Audiologia Clínica. São Paulo: Manole; 1999. p. 403-20.

15. Mcpherson D. Late Potentials of the auditory system. San Diego: Singular Publishing Group; 1996.
16. Musiek FE, Lee WW. Potenciais auditivos de média e longa latência. In: Musiek FE, Lee WW. Perspectivas atuais em avaliação auditiva. São Paulo: Manole; 2001. p. 239-67.

17. Durrant JD, Ferraro JA. Potenciais auditivos evocados de curta latência: eletrococleografia e audiometria de tronco encefálico. In: Musiek FE, Rintelmann WF. Perspectivas atuais em avaliação auditiva. São Paulo: Manole; 2001. cap. 8. p. 193-238.

18. Picton TW. The P300 wave of the human eventrelated potencial. Clin. Neurophysiol. 1992;9(1):456-79.

19. Gil R, Breux JP, Neau JP, Becq-Giraudon B, Pinon ML, Preut'Homme JL, Jouveaux T, Cazenave-Roblt F. Cognitive evoked potentials at the stages II and III of human immunodeficiency virus infection. Am. Med. Interne. 1991;142(4):297-302.

20. Fein G, Biggins CA, Mackay S. Delayed latency of the event-related brain potential P3A component in HIV disease. Progressive effects with increasing cognitive impairment. Arch. Neurol. 1996;53(8):715-6.

21. Polich J, Ilan A, Poceta JS, Mitler MM, Darko DF. Neuroelectric assessment of HIV: EEG, ERP, and viral load. Int. J. Psychophysiol. 2000;38(1):97-108.

22. Tartar JL, Sheehan C.M, Nash AJ, Starratt C, Puga A, Widmayer S. ERPs differ from neurometric tests in assessing HIV-associated cognitive deficit. Neuroreport. 2004;15(10): 1675-8.

23. Matas CG, Juan KR, Nakano RA. Potenciais evocados auditivos de média e longa latências em adultos com Aids. Pro Fono. 2006;18(2):171-6.

24. Birdsall HH, Ozluoglu LN, Lew HL, Trial J, Brown DP, Wofford MJ, Jerger JF, Rossen RD. Auditory P300 abnormalities and leukocyte activation in HIV infection. Otolaryngol. Head Neck Surg. 1994;110(2):53-9.

25. Chao LL, Lindgren JA, Flenniken DL, Wiener MW. ERP evidence of impaired central nervous system function in virally suppressed HIV patients on antiretroviral therapy. Clin. Neurophysil. 2004;115(7):1583-91.

26. Goodin DS, Aminoff MJ, Chernoff DN, Hollander H. Long latency event-related potentials in patients infected with human immunodeficiency virus. Ann. Neurol. 1990;27(4):414-9.

27. Galicia O, Sanchez-Alvez M, Diaz-Ruiz O, Narvaez FS, Elder J.H, Navarro L, Prospero-Garcia O. HIV derived protein gp120 supresses P3 potential in rats: potential implications in HIV associated dementia. Neuroreport. 2000;11(6):1351-5.

28. Chow KY, Ang LW, Verghesse I, Chew SK, Leo YS. Measurable predictive factors for progression to Aids among HIV-infected patients in Singapore. Ann. Acad. Med. 2005;34(1):84-9. 